\title{
Comparison of ab externo trabeculotomy in primary open-angle glaucoma and uveitic glaucoma: long-term outcomes
}

This article was published in the following Dove Press journal:

Clinical Ophthalmology

23 May 2016

Number of times this article has been viewed

\section{Antony William \\ Martin S Spitzer \\ Deshka Doycheva \\ Spyridon Dimopoulos \\ Martin Alexander Leitritz \\ Bogomil Voykov}

Centre for Ophthalmology, University Hospital Tübingen, Tübingen, Germany
Correspondence: Bogomil Voykov Centre for Ophthalmology, University Hospital Tübingen, Schleichstraße 12-16, 72076 Tübingen, Germany

Tel +49 707। 298372 I

Fax +49 707I 295455

Email bogomil.voykov@med.unituebingen.de
Background: The aim of this study was to compare the long-term outcomes of ab externo trabeculotomy in primary open-angle glaucoma (POAG) and uveitic glaucoma (UG).

Design: This was a retrospective single-center case series study.

Participants: Twenty eyes of 17 patients with POAG and 22 eyes of 18 patients with UG were included in this study.

Patients and methods: The medical records of all consecutive patients with POAG and UG who underwent ab externo trabeculotomy since 2004 were reviewed.

Main outcome measure: The main outcome measure was change in median intraocular pressure (IOP). Success was defined as IOP $\leq 21 \mathrm{mmHg}$ (success 1 ) and IOP $\leq 21 \mathrm{mmHg}$ and at least $25 \%$ reduction from baseline (success 2 ).

Results: In the POAG group, the median IOP decreased significantly from $22 \mathrm{mmHg}$ (95\% CI 21-25 mmHg; $\mathrm{n}=20)$ at baseline to $14 \mathrm{mmHg}(95 \%$ CI $12-16 ; \mathrm{n}=13)$ after 4 years, $P<0.001$. In the UG group, the median IOP decreased significantly from $27 \mathrm{mmHg}(95 \% \mathrm{CI}$ 24.5-30.5 mmHg; $\mathrm{n}=22)$ at baseline to $12 \mathrm{mmHg}(95 \%$ CI 9-15 mmHg; $\mathrm{n}=15)$ after 4 years, $P<0.001$. Seven eyes in the UG group failed within the first year after surgery compared to none in the POAG group. Of these, four eyes had Fuchs' uveitis syndrome and two had granulomatous uveitis. No sight-threatening complications occurred in both POAG and UG groups.

Conclusion: Ab externo trabeculotomy effectively reduced IOP in both UG and POAG groups. However, the success rates in the UG group were significantly lower due to the high failure rate in patients with Fuchs' uveitis syndrome and granulomatous uveitis. The procedure demonstrated a high safety profile in both UG and POAG patients.

Keywords: ab externo trabeculotomy, primary open-angle glaucoma, uveitic glaucoma

\section{Introduction}

Increased outflow resistance plays a major role in the elevation of intraocular pressure (IOP) in glaucoma. ${ }^{1}$ In primary open-angle glaucoma (POAG), increased outflow resistance is caused by increased extracellular material and modulation of trabecular meshwork (TM) cell tone by the action of the cells' actomyosin system in the juxtacanalicular tissue. ${ }^{2-4}$ In contrast, the elevation of IOP in uveitic glaucoma (UG) is caused by breakdown of the blood-ocular barrier followed by influx of proteins, immunocompetent cells, cytokines, and chemokines, which leads to obstruction of the $\mathrm{TM}$, direct tissue damage, and alteration in the function of the TM..$^{5-7}$ Recently, we have reported that ab externo trabeculotomy was moderately successful in UG after 3 years. ${ }^{8}$ However, the different mechanisms of IOP elevation in POAG and UG raise several questions. First, is there a difference in the efficacy of surgical procedures in POAG 
and UG? Second, if so, should we follow different surgical strategies in POAG and UG? Third, is chronic inflammation a risk factor for failure even if it was controlled preoperatively? There is only a limited number of studies that have compared a surgical procedure in both POAG and UG. ${ }^{9-12}$ None of them has reported on trabeculotomy. The purpose of our study was to compare the long-term results of ab externo trabeculotomy in patients with POAG and UG.

\section{Patients and methods}

We reviewed the medical records of all consecutive patients with POAG and UG who underwent ab externo trabeculotomy at the Centre for Ophthalmology at the University Hospital Tübingen since 2004. In both POAG and UG groups, the indication for surgery was insufficiently controlled IOP despite maximal tolerated medical treatment. Preoperatively, a target range IOP in the mid- to high-teens was considered sufficient based on age, glaucoma stage, visual field impairment, and tolerability for glaucoma medications in both POAG and UG groups. Ab externo trabeculotomy was performed in patients who were considered to have a high risk of bleb failure or bleb-related complications due to chronic conjunctival inflammation or young age. We documented age at surgery, sex, best-corrected visual acuity, IOP, number of glaucoma medications, and postoperative complications for each patient. Success was defined as IOP $\leq 21 \mathrm{mmHg}$ (success 1 ) and IOP $\leq 21 \mathrm{mmHg}$ and at least $25 \%$ reduction from baseline (success 2 ). The success was complete if after glaucoma surgery no further glaucoma medication was required and qualified if glaucoma medication or a cycloablative procedure was necessary to control IOP. If the IOP was $>21 \mathrm{mmHg}$ at two consecutive visits despite reintroduction of glaucoma medications and further surgical treatment such as filtering surgery or the implantation of Ahmed glaucoma valve necessary to control IOP, then these eyes were considered as failure. The examination before the reoperation was then the final follow-up, and IOP data were displaced forward. A minimum follow-up of 3 years was required for inclusion.

This work adhered to the tenets of the Declaration of Helsinki. According to the requirements of the institutional review board of Eberhard Karls University of Tuebingen, as completely anonymized data were used for this study institutional review board approval was not required. Patients signed an informed consent prior to surgery, but the use of anonymized retrospective data does not require additional consent of the patient according to the requirements of the IRB.

\section{Operative technique}

The surgical procedure has been described previously. ${ }^{13}$ Briefly, after conjunctival incision, a $4 \mathrm{~mm} \times 4 \mathrm{~mm}$ scleral flap was created at the corneal limbus. The Schlemm's canal was identified and deroofed. U-shaped probes were then inserted into both ends of the opened canal and rotated $90^{\circ}$ against the TM. Rotation of the probes achieved at least $120^{\circ}$ opening of the TM. The scleral flap was then closed watertight. Postoperative treatment comprised topical administration of combined antibiotic and steroid medication for 2 weeks.

Pilocarpine $1 \%$ was given postoperatively for 4 weeks to avoid formation of peripheral anterior synechia. In the UG group, uveitis was inactive in all eyes before surgery for at least 3 months and all patients preoperatively received systemic body weight-adjusted oral steroid therapy $(1 \mathrm{mg} / \mathrm{kg})$ starting 5 days prior to surgery. Steroids were then tapered over 10-12 weeks after surgery.

\section{Statistical analysis}

For statistical analysis, chi-squared test, one-way analysis of variance, and Fisher's exact test were performed using JMP software (version 11.0; SAS Institute Inc., Cary, NC, USA). $P$-values $<0.05$ were considered to reflect significant differences.

\section{Results}

Twenty eyes of 17 patients with POAG and 22 eyes of 18 patients with UG were included in this study. The demographic characteristics of the patients are summarized in Table 1. Uveitic etiology included idiopathic anterior uveitis (six eyes), idiopathic intermediate uveitis (four eyes), posterior uveitis (two eyes), Fuchs' uveitis syndrome (FUS; five eyes), herpetic uveitis (one eye), Posner-Schlossman syndrome (two eyes), and granulomatous uveitis (two eyes).

Table I Patients' demographics

\begin{tabular}{|c|c|c|c|}
\hline Demographic & POAG & UG & $P$-value \\
\hline Eyes, $\mathrm{n}$ & 20 & 22 & 0.6 \\
\hline \multicolumn{4}{|l|}{ Sex } \\
\hline Female, n (\%) & $17(85)$ & $4(18)$ & \\
\hline Male, n (\%) & $3(15)$ & $18(82)$ & \\
\hline \multicolumn{4}{|l|}{ Age (years) } \\
\hline Mean \pm SD & $62.5 \pm 9.7$ & $38 \pm 20.7$ & $<\mathbf{0 . 0 0 I}$ \\
\hline Range & $40-76$ & II-74 & \\
\hline \multicolumn{4}{|c|}{ Baseline IOP (mmHg) } \\
\hline Median & 22 & 27 & 0.02 \\
\hline $95 \% \mathrm{Cl}$ & $21.4-25.2$ & $24.5-30.5$ & \\
\hline \multicolumn{4}{|c|}{ Baseline medications } \\
\hline Median & 4 & 5 & $<0.001$ \\
\hline $95 \% \mathrm{Cl}$ & $3.2-4.0$ & $4.2-5$ & \\
\hline \multicolumn{4}{|c|}{ Baseline BCVA (logMAR) } \\
\hline Median & 0.2 & 0.5 & $<0.001$ \\
\hline $95 \% \mathrm{Cl}$ & $0.05-0.5$ & $0.4-0.8$ & \\
\hline
\end{tabular}

Note: Statistically significant changes are shown in bold in the $P$-value column. Abbreviations: POAG, primary open-angle glaucoma; UG, uveitic glaucoma; $I O P$, intraocular pressure; BCVA, best-corrected visual acuity; logMAR, logarithm of the minimum angle of resolution; $\mathrm{Cl}$, confidence interval. 
Uveitis was inactive for at least 3 months in all the patients preoperatively.

In four eyes with anterior uveitis, uveitis was controlled with methotrexate and in two eyes with adalimumab and mycophenolate mofetil. In the eyes with intermediate uveitis, inflammation was controlled with mycophenolate sodium in two eyes, mycophenolate mofetil in one eye, and low-dose systemic steroid in another eye. Patients with posterior uveitis received low-dose systemic steroids. All five patients with FUS received only nonsteroid anti-inflammatory eyedrops. Herpetic uveitis was controlled with systemic acyclovir. In the two eyes with Posner-Schlossman syndrome, uveitis was controlled with valganciclovir. Granulomatous uveitis was controlled with methotrexate.

Postoperative reactivation of the uveitis was not seen in any patient during the follow-up.

\section{IOP and glaucoma medications}

The median IOP in the POAG group decreased significantly from $22 \mathrm{mmHg}(95 \%$ CI 21-25 mmHg; $\mathrm{n}=20)$ at baseline to $14 \mathrm{mmHg}(95 \% \mathrm{CI} 12-15 \mathrm{mmHg} ; \mathrm{n}=20, P<0.001)$ after 3 years and remained $14 \mathrm{mmHg}(95 \%$ CI $12-16 \mathrm{mmHg}$; $\mathrm{n}=13, P<0.001)$ after 4 years. Similarly, the median IOP in the UG group decreased from $27 \mathrm{mmHg}(95 \% \mathrm{CI}$ 24.5-30.5 mmHg; $\mathrm{n}=22)$ at baseline to $15 \mathrm{mmHg}(95 \% \mathrm{CI}$ $13-16 \mathrm{mmHg} ; \mathrm{n}=22, P<0.001)$ after 3 years and to $12 \mathrm{mmHg}$ (95\% CI 9-15 mmHg; n=15, $P<0.001$ ) after 4 years. The median IOP was statistically significantly different between the UG group and POAG group at baseline $(P=0.03)$ on the first postoperative day $(P<0.01)$ and at the 1 -year follow-up $(P=0.01)$. Figure 1 shows the median IOP at different time points in both groups.

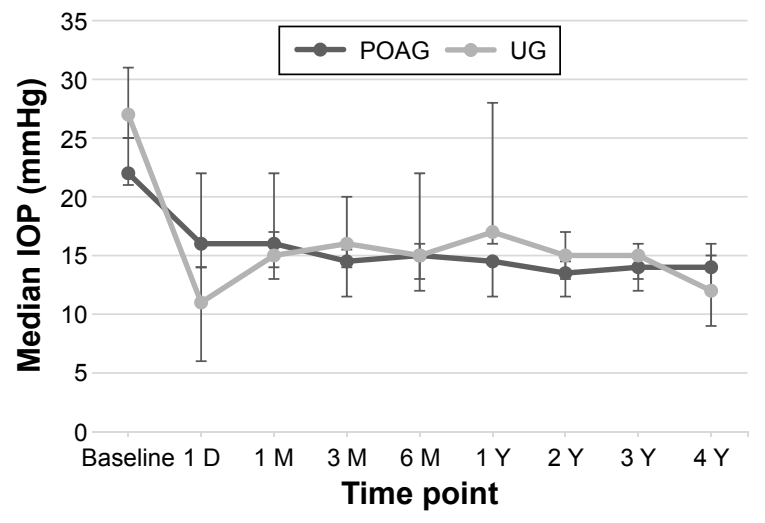

Figure I Median IOP at different time points.

Notes: Decrease in median IOP was statistically significant for all time points compared to baseline value in both groups. The whiskers represent the $95 \% \mathrm{Cl}$. The median IOP was statistically significantly different between the UG group and POAG group at baseline $(P=0.03)$, on the first postoperative day $(P<0.0 \mathrm{I})$, and at the I-year follow-up $(P=0.01)$.

Abbreviations: IOP, intraocular pressure; UG, uveitic glaucoma; POAG, primary open-angle glaucoma; D, day; $M$, month; $Y$, year.
In the POAG group, the median number of glaucoma medications decreased significantly from 4 (95\% CI 3.2-4; $\mathrm{n}=20)$ at baseline to $2(95 \%$ CI $1.4-2.6 ; \mathrm{n}=20, P<0.001)$ after 3 years and remained 2 (95\% CI 1.0-2.4; $\mathrm{n}=13, P<0.001)$ after 4 years. The median number of glaucoma medications in the UG group decreased significantly from 5 (95\% CI 4.2-5.0; $\mathrm{n}=22)$ at baseline to $1(95 \% \mathrm{CI} 0.5-1.7 ; \mathrm{n}=22, P<0.001)$ after 3 years and remained 1 (95\% CI $0.3-2.3 ; n=15, P<0.001)$ after 4 years. The median number of glaucoma medications showed no statistically significant differences between both groups at the different time points.

Cycloablative treatment was performed once within the first 6 months in three eyes and repeated in a single eye within the first year postoperatively in the UG group. Only one eye received cycloablative treatment 1 week postoperatively in the POAG group.

Seven eyes failed in the UG group compared to none in the POAG group. Failure in the UG group occurred within the first 3 months in six eyes and after 7 months in one eye. In all these eyes, Ahmed glaucoma valve was needed to control the IOP. Success results are shown in Table 2.

\section{Complications}

Hyphema was the most common postoperative complication in both groups. It resolved completely within 4 weeks postoperatively without any interventions. We did not observe any sight-threatening complications during the 4 years of follow-up. Complications are presented in Table 3.

\section{Discussion}

There is only a limited number of studies that have compared the outcomes of a surgical procedure between patients with POAG and patients with UG. ${ }^{9-12}$ However, since the pathomechanisms of increased IOP differ between UG and POAG, it is important to know if a surgical procedure can achieve similar results in both conditions.

$\mathrm{Ab}$ externo trabeculotomy is usually a first-choice surgical treatment in congenital and juvenile glaucoma. However, a study by Tanihara et $\mathrm{al}^{13}$ has demonstrated that the procedure is an effective treatment for POAG, as well, even in adults. In this study, the success rate of the procedure was $58 \%$ after 5 years. The authors also reported that the success was significantly higher in patients with lower preoperative IOP $(<30 \mathrm{mmHg})$. In these patients, the final success probability after 5 years was $66.9 \%$. On the other hand, age did not significantly influence the success of surgery. Although direct comparison is difficult because of different study populations and success criteria, our results in the POAG group are similar (qualified success). However, the success 
Table 2 Success rates at different time points

\begin{tabular}{|c|c|c|c|c|c|c|c|c|}
\hline \multirow[t]{2}{*}{ Success } & \multicolumn{2}{|l|}{ I year } & \multicolumn{2}{|l|}{2 years } & \multicolumn{2}{|l|}{3 years } & \multicolumn{2}{|l|}{4 years } \\
\hline & $\begin{array}{l}\text { POAG } \\
(n=20)\end{array}$ & $\begin{array}{l}\text { UG } \\
(n=22)\end{array}$ & $\begin{array}{l}\text { POAG } \\
(n=20)\end{array}$ & $\begin{array}{l}\text { UG } \\
(n=22)\end{array}$ & $\begin{array}{l}\text { POAG } \\
(n=20)\end{array}$ & $\begin{array}{l}\text { UG } \\
(n=22)\end{array}$ & $\begin{array}{l}\text { POAG } \\
(n=13)\end{array}$ & $\begin{array}{l}\text { UG } \\
(n=15)\end{array}$ \\
\hline \multicolumn{9}{|l|}{ Complete success } \\
\hline$\leq 21 \mathrm{mmHg}, \mathrm{n}(\%)$ & $4(20)$ & $4(18)$ & $5(25)$ & $3(14)$ & $4(20)$ & $5(23)$ & $2(15)$ & $2(13)$ \\
\hline$P$-value & 1.0 & & 0.44 & & 1.0 & & 1.0 & \\
\hline$\leq 21 \mathrm{mmHg}$ and $\geq 25 \%$ IOP reduction from baseline, $\mathrm{n}(\%)$ & $4(20)$ & $4(18)$ & $2(10)$ & $3(14)$ & $2(10)$ & $5(23)$ & I (8) & $3(20)$ \\
\hline$P$-value & 1.0 & & 1.0 & & 0.44 & & 0.6 & \\
\hline \multicolumn{9}{|l|}{ Qualified success } \\
\hline$\leq 21 \mathrm{mmHg}, \mathrm{n}(\%)$ & $16(80)$ & II (50) & $15(75)$ & $12(55)$ & $16(80)$ & $10(45)$ & II (85) & $6(40)$ \\
\hline$P$-value & 0.02 & & 0.12 & & 0.03 & & 0.03 & \\
\hline$\leq 21 \mathrm{mmHg}$ and $\geq 25 \%$ IOP reduction from baseline, $\mathrm{n}(\%)$ & $12(60)$ & $8(36)$ & $15(75)$ & $9(4 I)$ & $14(70)$ & $7(32)$ & $8(62)$ & $4(27)$ \\
\hline$P$-value & 0.2 & & 0.03 & & 0.03 & & 0.12 & \\
\hline \multicolumn{9}{|l|}{ Failure } \\
\hline n (\%) & None & $7(32)$ & None & $7(32)$ & None & $7(32)$ & None & $7(47)$ \\
\hline
\end{tabular}

Notes: Success was defined as IOP $\leq 2 \mathrm{I} \mathrm{mmHg}$ (success I) and IOP $\leq 2 \mathrm{I} \mathrm{mmHg}$ and at least $25 \%$ reduction from baseline (success 2 ). Complete success was achieved when no further glaucoma medication was required after glaucoma surgery. If glaucoma medication or a cycloablative procedure was necessary to control IOP, then success was qualified. If the IOP was $>21 \mathrm{mmHg}$ at two consecutive visits despite reintroduction of glaucoma medications and further surgical treatment such as filtering surgery or the implantation of Ahmed glaucoma valve was necessary to control IOP, then these eyes were considered as failure. Statistically significant changes are shown in bold.

Abbreviations: POAG, primary open-angle glaucoma; UG, uveitic glaucoma; IOP, intraocular pressure.

rate in the UG group was significantly lower. The different pathomechanisms of IOP elevation in POAG and UG groups are a possible explanation for this difference. This is demonstrated by the fact that the majority of failures in the UG group occurred in patients with FUS (four eyes) and granulomatous uveitis (two eyes). Typically, the inflammation is of low grade, but more difficult to control and irresponsive to steroids in patients with FUS compared to other uveitis forms. ${ }^{14} \mathrm{~A}$ recent study described the intraoperative observation of a perilimbal and/or episcleral vessel fluid wave seen during trabectome surgery and termed it episcleral venous fluid wave. ${ }^{15}$ The episcleral venous fluid wave correlated well with the outcomes of trabectome surgery in patients with various types of open-angle glaucomas. The authors demonstrated that if a wave was not seen or barely present, there was likely obstruction in the collector channel opening or intrascleral channels preventing flow to the episcleral veins. ${ }^{16}$ Unfortunately, the authors did not report on different subtypes of glaucomas. However, it is possible that the persistent low-grade inflammation in FUS leads to obstruction in the collector channel opening or the intrascleral channels, which could explain the high failure rate in FUS eyes in

Table 3 Postoperative complications

\begin{tabular}{lll}
\hline Complication & POAG, $\mathbf{n}(\%)$ & UG, $\mathbf{n}(\%)$ \\
\hline Hyphema & II (55) & $7(32)$ \\
Hypotony & $\mathrm{I}(5)$ & $\mathrm{I}(5)$ \\
Filtering bleb & $\mathrm{I}(5)$ & $\mathrm{I}(5)$ \\
Vitreous prolapse & 0 & $\mathrm{I}(5)$ \\
\hline
\end{tabular}

Abbreviations: POAG, primary open-angle glaucoma; UG, uveitic glaucoma. our study. Similarly, Hamanaka et $\mathrm{al}^{17}$ showed that eyes with granulomatous uveitis are associated with infiltration of lymphocytes, monocytes, and macrophages around the inner wall, posterior outer wall of the Schlemm's canal, and the collector channels, leading to narrowing and occlusion of the Schlemm's canal. Interestingly, a recent histological study suggested that the enhancement of conventional routes may not, however, be important for the IOP-lowering effect of trabeculotomy. The authors hypothesized that four different types of histological changes in the TM may reduce enhancement of the newly created unconventional routes by trabeculotomy.$^{18}$ In this case, it is possible that in UG, the breakdown of the blood-ocular barrier followed by influx of proteins, immunocompetent cells, cytokines, and chemokines leads more often to the aforementioned histological changes in the TM compared to POAG. Again, active inflammation in FUS probably accelerates these changes even more. Consequently, pre- and postoperative care is crucial for improving the success rate of trabeculotomy in uveitis patients. In our experience, surgery should be performed in eyes that have been calm for at least 3 months prior to surgery. Preoperatively, we treat the patients with systemic body weight-adjusted steroid therapy, which is started 5 days before surgery $(1 \mathrm{mg} / \mathrm{kg})$. We then taper the steroids slowly during a period of 10-12 weeks postoperatively to avoid recurrence of inflammation.

Notably, the outcomes of filtration surgery are also worse in UG compared to POAG. Kaburaki et $\mathrm{al}^{10}$ reported similar success rates after 5 years of follow-up in a retrospective study in patients with inactive uveitis in comparison to 
patients with POAG. However, postoperative complications such as long-standing ocular hypotony, hypotonic maculopathy, and cataract formation were more common in the UG group. In contrast, Noble et $\mathrm{al}^{11}$ reported that results of trabeculectomy with MMC were significantly worse in patients with UG compared to patients with POAG. UG patients required also more postoperative procedures and developed more often cystic blebs. Additionally, a study by Iwao et $\mathrm{al}^{9}$ demonstrated that trabeculectomy with MMC was less effective in maintaining the IOP reduction in UG patients than in POAG patients. One study compared the results of Ahmed glaucoma valve implantation between patients with UG and patients with POAG. The success rates were similar in both groups after 30 months. However, failure in terms of tube removal occurred significantly more often in patients with UG. ${ }^{12}$

The role of the higher preoperative IOP as a prognostic factor associated with poor prognosis after ab externo trabeculotomy remains a matter of debate. Tanihara et al ${ }^{13}$ reported that higher preoperative IOP was a negative prognostic factor in POAG. In contrast, Iwao et al ${ }^{19}$ demonstrated that higher preoperative IOP was not a prognostic factor for surgical failure of trabeculotomy in the treatment of steroid-induced glaucoma. Likewise, in our study, the preoperative IOP in the eyes with failure was not statistically significant different compared to the eyes without failure. However, due to the small number of eyes, we could not determine the role of preoperative IOP level conclusively.

The influence of age on the outcomes of ab externo trabeculotomy has been controversially discussed in the literature. Some authors have reported better results in patients with congenital and juvenile glaucoma compared to those with adult-onset glaucoma. ${ }^{20,21}$ However, Tanihara et a ${ }^{13}$ showed that there was no significant difference in the outcomes between a younger ( $<60$ years) and an elderly ( $\geq 60$ years) group having eyes with POAG. In our study, patients with UG were much younger than those with POAG. However, in our opinion, the worse results in the aforementioned studies by Schwartz and Anderson, Lunt and Livingston, and ours for the non-POAG eyes compared to the eyes with POAG are caused by structural changes of the outflow system that differ among various glaucoma types and are only indirectly correlated to age.

There was a statistically significant difference in sex between both groups in our study. However, the failure rates were not statistically significantly different in both sexes, and sex was not a prognostic factor for success or failure. The strength of this analysis is limited by the small number of patients. Surprisingly, the influence of sex on the outcomes of glaucoma surgery has not yet been specifically addressed in the published literature. This makes it impossible for us to compare our results to others.

A major advantage of ab externo trabeculotomy is that it avoids the potentially sight-threatening complications of filtration surgery. In our study, complication rates were similar and low in both UG and POAG groups, although postoperative hyphema was slightly more common in the POAG group. Interestingly, Grieshaber et $\mathrm{al}^{22}$ demonstrated that postoperative hyphema was a positive prognostic indicator in uneventful canaloplasty in regard to IOP reduction, possibly representing a restored and patent physiologic aqueous outflow system.

The limitations of our study include its retrospective design as well as the variety of UG types treated. Notably, the outcomes in eyes with FUS and granulomatous uveitis were worse compared to both the remaining eyes in the UG group and the POAG group. However, the small number of eyes precluded from further analyses of these differences.

\section{Conclusion}

Our results showed that ab externo trabeculotomy effectively reduced IOP in both UG and POAG groups. However, the long-term success rates in the UG group were significantly lower due to the high failure rate in patients with FUS and granulomatous uveitis. The procedure demonstrated a high safety profile in both UG and POAG patients.

\section{Disclosure}

The authors report no conflicts of interest in this work.

\section{References}

1. Gabelt BT, Kaufman PL. Changes in aqueous humor dynamics with age and glaucoma. Prog Retin Eye Res. 2005;24(5):612-637.

2. Johnson M, Chan D, Read AT, Christensen C, Sit A, Ethier CR. The pore density in the inner wall endothelium of Schlemm's canal of glaucomatous eyes. Invest Ophthalmol Vis Sci. 2002;43(9):2950-2955.

3. Lutjen-Drecoll E. Functional morphology of the trabecular meshwork in primate eyes. Prog Retin Eye Res. 1999;18(1):91-119.

4. Tamm ER. The trabecular meshwork outflow pathways: structural and functional aspects. Exp Eye Res. 2009;88(4):648-655.

5. Deuter CM, Klinik T, Muller M, Geerling G, Zierhut M. Sekundärglaukom bei Uveitis [Secondary glaucoma in uveitis]. Ophthalmologe. 2010;107(5):427-434. German

6. Moorthy RS, Mermoud A, Baerveldt G, Minckler DS, Lee PP, Rao NA. Glaucoma associated with uveitis. Surv Ophthalmol. 1997;41(5):361-394.

7. Sung VC, Barton K. Management of inflammatory glaucomas. Curr Opin Ophthalmol. 2004;15(2):136-140.

8. Voykov B, Dimopoulos S, Leitritz MA, Doycheva D, William A. Longterm results of ab externo trabeculotomy for glaucoma secondary to chronic uveitis. Graefes Arch Clin Exp Ophthalmol. 2016;254(2):355-360. 
9. Iwao $\mathrm{K}$, Inatani M, Seto $\mathrm{T}$, et al. Long-term outcomes and prognostic factors for trabeculectomy with mitomycin $\mathrm{C}$ in eyes with uveitic glaucoma: a retrospective cohort study. J Glaucoma. 2014;23(2):88-94.

10. Kaburaki T, Koshino T, Kawashima H, et al. Initial trabeculectomy with mitomycin $\mathrm{C}$ in eyes with uveitic glaucoma with inactive uveitis. Eye (Lond). 2009;23(7):1509-1517.

11. Noble J, Derzko-Dzulynsky L, Rabinovitch T, Birt C. Outcome of trabeculectomy with intraoperative mitomycin $\mathrm{C}$ for uveitic glaucoma. Can J Ophthalmol. 2007;42(1):89-94.

12. Rachmiel R, Trope GE, Buys YM, Flanagan JG, Chipman ML. Ahmed glaucoma valve implantation in uveitic glaucoma versus open-angle glaucoma patients. Can J Ophthalmol. 2008;43(4):462-467.

13. Tanihara H, Negi A, Akimoto M, et al. Surgical effects of trabeculotomy ab externo on adult eyes with primary open angle glaucoma and pseudoexfoliation syndrome. Arch Ophthalmol. 1993;111(12):1653-1661.

14. Mohamed Q, Zamir E. Update on Fuchs' uveitis syndrome. Curr Opin Ophthalmol. 2005;16(6):356-363.

15. Fellman RL, Grover DS. Episcleral venous fluid wave: intraoperative evidence for patency of the conventional outflow system. J Glaucoma. 2014;23(6):347-350.
16. Fellman RL, Feuer WJ, Grover DS. Episcleral venous fluid wave correlates with trabectome outcomes: intraoperative evaluation of the trabecular outflow pathway. Ophthalmology. 2015;122(12):2385e-2391e.

17. Hamanaka T, Takei A, Takemura T, Oritsu M. Pathological study of cases with secondary open-angle glaucoma due to sarcoidosis. Am J Ophthalmol. 2002;134(1):17-26.

18. Amari Y, Hamanaka T, Futa R. Pathologic investigation failure of trabeculotomy. J Glaucoma. 2015;24(4):316-322.

19. Iwao K, Inatani M, Tanihara H; Japanese Steroid-Induced Glaucoma Multicenter Study Group. Success rates of trabeculotomy for steroidinduced glaucoma: a comparative, multicenter, retrospective cohort study. Am J Ophthalmol. 2011;151(6):1047e-1056e.

20. Luntz MH, Livingston DG. Trabeculotomy ab externo and trabeculectomy in congenital and adult-onset glaucoma. Am J Ophthalmol. 1977; 83(2):174-179.

21. Schwartz AL, Anderson DR. Trabecular surgery. Arch Ophthalmol. 1974; 92(2):134-138.

22. Grieshaber MC, Schoetzau A, Flammer J, Orgul S. Postoperative microhyphema as a positive prognostic indicator in canaloplasty. Acta Ophthalmol. 2013;91(2):151-156.
Clinical Ophthalmology

\section{Publish your work in this journal}

Clinical Ophthalmology is an international, peer-reviewed journal covering all subspecialties within ophthalmology. Key topics include: Optometry; Visual science; Pharmacology and drug therapy in eye diseases; Basic Sciences; Primary and Secondary eye care; Patient Safety and Quality of Care Improvements. This journal is indexed on

\footnotetext{
Submit your manuscript here: http://www.dovepress.com/clinical-ophthalmology-journal
}

\section{Dovepress}

PubMed Central and CAS, and is the official journal of The Society of Clinical Ophthalmology (SCO). The manuscript management system is completely online and includes a very quick and fair peer-review system, which is all easy to use. Visit http://www.dovepress.com/ testimonials.php to read real quotes from published authors. 